\title{
Radiographic growth indicators: The issue of diagnostic reliability and clinical feasibility
}

\author{
Perinetti, Giuseppe *; Contardo, Luca * \\ * Department of Medical, Surgical and Health Sciences, School of Dentistry, University of Trieste, Trieste, Italy
}

\begin{abstract}
The use of radiographic growth indicators, such as the hand-and-wrist maturation (HWM) and cervical vertebral maturation (CVM) methods, has been recommended in previous clinical trials on the skeletal effects obtained by functional treatment in growing Class II patients. The concept behind the use of indicators resides in the identification of the pubertal growth spurt in individual patients allowing the delivery of the treatment at this specific stage of skeletal maturation when the mandible response is expected to be maximum growth. Interestingly, while many of the former types of investigations reported a poor correlation between the stages of growth indicators (mainly the CVM) and mandibular growth spurt, most of the latter type of investigations reported clinically relevant favorable effects when the growth indicators are used. It is perhaps that investigations are still missing the relevant piece of evidence. The first critical issue relates to the correlation between two variables (i.e., stages of maturation and mandibular growth) that does not imply diagnostic accuracy, as it has been extensively reported for the case of dental maturation. The other relevant issue relates to the clinical feasibility of the repetition of the recording when dealing with invasive methods based on X-rays, irrespective of whether the methods are accurate. Meanwhile, more opportunity will be given by the use of non-invasive (serum or GCF) biomarkers. According to all these considerations, more reports will be necessary to elucidate the role of the growth indicators in orthodontics fully.
\end{abstract}

Perinetti G, Contardo L. Radiographic growth indicators: The issue of diagnostic reliability and clinical feasibility. South Eur J Orthod Dentofac Res. 2017;4(2):48-49.

The use of radiographic growth indicators, such as the handand-wrist maturation (HWM) and cervical vertebral maturation (CVM) methods, has been recommended in previous clinical trials on the skeletal effects obtained by functional treatment in growing Class II patients. ${ }^{1}$ These indicators are based on the attainment of discrete stages according to pre-established morphological maturational features. The concept behind the use of indicators resides in the identification of the pubertal growth spurt in individual patients allowing the delivery of the treatment at this specific stage of skeletal maturation when the mandible response is expected to be maximum growth. ${ }^{2}$ However, contrasting recommendations have been carried out from current research regarding the reliability of such

\section{Corresponding Author:}

Giuseppe Perinetti,

Struttura Complessa di Clinica Odontoiatrica e Stomatologica, Ospedale Maggiore,

Piazza Ospitale 1, 34129 Trieste, Italy.

e-mail: G.Perinetti@fmc.units.it methods. ${ }^{3,4}$ Studies on the radiographical growth indicators are essentially of two types: the ones correlating the various stages of maturation with mandibular growth spurt, ${ }^{5}$ and the ones evaluating skeletal effects obtained by functional treatment in Class II patients the timing of treatment has been based on such indicators (for review, see Perinetti et al. ${ }^{4}$ ) Interestingly, while many of the former types of investigations reported poor correlation between the stages of growth indicators (mainly the CVM) and mandibular growth spurt, ${ }^{6}$ most of the latter type of investigations reported clinically relevant favourable effects when the growth indicators are used. ${ }^{4}$ It is perhaps that investigations are still missing the relevant piece of evidence.

The first critical issue relates to the correlation between two variables (i.e., stages of maturation and mandibular growth) that does not imply diagnostic accuracy, as it has been extensively reported for the case of dental maturation. ${ }^{7}$ According to this point of view, the latter parameter is the one of great clinical importance. However, in spite of this concept, to date, only two studies have focused explicitly on the diagnostic accuracy of the $\mathrm{CVM}^{8}$ and middle phalanx maturation (MPM) ${ }^{9}$ methods in the identification of the mandibular growth peak. Therefore, relevant data has still to be reported on the diagnostic capability 
of the different methods in the identification of the mandibular growth spurt in individual patients. In this context, it is worth of mentioning that huge and expensive clinical trials (even randomised trials $s^{10,11}$ ) have been performed basing the timing of intervention on diagnostic methods for which the authors did not even know the diagnostic accuracy.

The other relevant issue relates to the clinical feasibility of the repetition of the recording when dealing with invasive methods based on X-rays, irrespective of whether the methods are accurate. It has been reported that the length of the different stages of a growth indicator may be subjected to noteworthy variation, ${ }^{12}$ rendering the precise identification of the timing of intervention reliable only if a longitudinal monitoring is followed from a pre-pubertal stage of development to the pubertal one. This aspect may strongly limit the use of radiographical indicators, such as the HWM and CVM methods, as the procedure of repeated recordings is harmful to the patient. ${ }^{13}$ Meanwhile, more opportunity will be given by the use of noninvasive (serum or GCF) biomarkers, ${ }^{14}$ the monitoring of the sole third finger middle phalanx maturation ${ }^{9}$ may be a valid compromise between the necessity of longitudinal recordings and acceptable radiation exposure to the patient.

According to all these considerations, more reports will be necessary to fully elucidate the role of the growth indicators in orthodontics and how their use may enhance the efficiency of the functional treatments.

\section{REFERENCES}

1. Perinetti G, Contardo L. Reliability of Growth Indicators and Efficiency of Functional Treatment for Skeletal Class II Malocclusion: Current Evidence and Controversies. Biomed Res Int. 2017;2017:1367691.

2. McNamara JA, Jr, Brudon WL. Orthodontics and dentofacial orthopedics. Ann Arbor: Needham Press; 2001.

3. Thiruvenkatachari B, Harrison JE, Worthington HV, O'Brien KD. Orthodontic treatment for prominent upper front teeth (Class II malocclusion) in children. Cochrane Database Syst Rev. 2013:(11):CD003452.

4. Perinetti G, Primožič J, Franchi L, Contardo L. Treatment Effects of Removable Functional Appliances in Pre-Pubertal and Pubertal Class II Patients: A Systematic Review and Meta-Analysis of Controlled Studies. PLoS One. 2015 Oct 28;10(10):e0141198.

5. Alkhal HA, Wong RW, Rabie AB. Correlation between chronological age, cervical vertebral maturation and Fishman's skeletal maturity indicators in southern Chinese. Angle Orthod. 2008;78(4):591-6.

6. Gray S, Bennani H, Kieser JA, Farella M. Morphometric analysis of cervical vertebrae in relation to mandibular growth. Am J Orthod Dentofacial Orthop. 2016;149(1):92-8.

7. Perinetti G, Westphalen GH, Biasotto M, Salgarello S, Contardo L. The diagnostic performance of dental maturity for identification of the circumpubertal growth phases: a meta-analysis. Prog Orthod. 2013;14:8.

8. Perinetti G, Contardo L, Castaldo A, McNamara JA, Jr., Franchi L. Diagnostic reliability of the cervical vertebral maturation method and standing height in the identification of the mandibular growth spurt. Angle Orthod. 2016;86(4):599-609.

9. Perinetti G, Sbardella V, Contardo L. Diagnostic reliability of the third finger middle phalanx maturation (MPM) method in the identification of the mandibular growth peak. Eur J Orthod. 2017;39(2):194-201.

10. Tulloch JF, Phillips C, Koch G, Proffit WR. The effect of early intervention on skeletal pattern in Class II malocclusion: a randomized clinical trial. Am J Orthod Dentofacial Orthop. 1997;111(4):391-400.

11. O’Brien K, Wright J, Conboy F, Sanjie Y, Mandall N, Chadwick S, et al. Effectiveness of early orthodontic treatment with the Twin-block appliance: a multicenter, randomized, controlled trial. Part 1: Dental and skeletal effects. Am J Orthod Dentofacial Orthop. 2003;124(3):234-43.

12. Perinetti G, Bianchet A, Franchi L, Contardo L. Cervical vertebral maturation: An objective and transparent code staging system applied to a 6-year longitudinal investigation. Am J Orthod Dentofacial Orthop. 2017;151(5):898-906.

13. Patcas R, Signorelli L, Peltomäki T, Schätzle M. Is the use of the cervical vertebrae maturation method justified to determine skeletal age? A comparison of radiation dose of two strategies for skeletal age estimation. Eur J Orthod. 2013;35(5):604-9.

14. de Aguiar MC, Perinetti G, Capelli J Jr. The Gingival Crevicular Fluid as a Source of Biomarkers to Enhance Efficiency of Orthodontic and Functional Treatment of Growing Patients. Biomed Res Int. 2017;2017:3257235. 